\title{
Aa. Vv., Essai et essayisme en France au XIX siècle, sous la direction de Pierre Glaudes et Boris Lyon-Caen
}

\section{Maria Emanuela Raffi}

\section{OpenEdition}

\section{Journals}

\section{Edizione digitale}

URL: http://journals.openedition.org/studifrancesi/567

DOI: 10.4000/studifrancesi.567

ISSN: 2421-5856

\section{Editore}

Rosenberg \& Sellier

\section{Edizione cartacea}

Data di pubblicazione: 1 aprile 2015

Paginazione: 183-184

ISSN: 0039-2944

\section{Notizia bibliografica digitale}

Maria Emanuela Raffi, « Aa. VV., Essai et essayisme en France au xix siècle, sous la direction de Pierre Glaudes et Boris Lyon-Caen », Studi Francesi [Online], 175 (LIX | I) | 2015, online dal 01 avril 2015, consultato il 18 septembre 2020. URL : http://journals.openedition.org/studifrancesi/567 ; DOI : https://doi.org/10.4000/studifrancesi.567

Questo documento è stato generato automaticamente il 18 settembre 2020.

\section{(c) (i) (9)}

Studi Francesi è distribuita con Licenza Creative Commons Attribuzione - Non commerciale - Non opere derivate 4.0 Internazionale. 


\title{
Aa. Vv., Essai et essayisme en France au XIXe siècle, sous la direction de Pierre Glaudes et Boris Lyon-Caen
}

\author{
Maria Emanuela Raffi
}

\section{NOTIZIA}

Aa. Vv., Essai et essayisme en France au XIX siècle, sous la direction de Pierre GLAUDES et Boris LYON-CAEN, Paris, Classiques Garnier, 2014, pp. 291.

1 "Genre-carrefour», una definizione dell'essai è tutt'altro che semplice; è ciò che sottolineano i due curatori nell'«Introduction» al volume, indicando i criteri di una possibile classificazione per il «texte essayiste»: in base alla forma (dalla «note fragmentaire» al saggio strutturato), al tipo di rapporto con il testo di riferimento, alla presenza e allo statuto del «personnage conceptuel» e infine in base al tipo di scrittura, allo stile.

2 La prima delle cinque parti del volume («Essai et formes connexes dans la première moitié du siècle») inizia con lo studio di étienne BEAULIEU su Joseph Joubert, La pensée inachevée. Composta da saggi incompiuti e mai pubblicati - raccolti poi nei Carnets da André Beaunier -, l'opera di Joubert appare all'A. come l'esempio privilegiato di una certa visione romantica, refrattaria ad ogni sistematicità, tenacemente monarchica e ostile alla voga nascente del romanzo, contenitore di «passions désordonnées».

3 Con Le goût $d u$ composite di I rène LANGLET ci si sposta nell'ambito della saggistica gastronomica con un'analisi della Physiologie du Goût di Brillat-Savarin, già nobilitato dalla prefazione di Roland Barthes nel 1975. Partendo dal commento di Barthes ed esaminando le radici inglesi del genere e la presenza costante dell'humour, l'A. arriva ad avvicinare il saggio al genere della causerie, genere anch'esso composito, fatto «de récits, d'anecdotes, de préceptes, de bons mots» e in questo caso anche di ricette di cucina. 
4 Alle numerose Physiologies pubblicate negli anni 1840 è dedicato lo studio di valérie STIÉNON Défaire le discours d'autorité, in cui vengono messi in luce i tratti caratteristici del «genre physiologique»: «l'influence londonienne» con frequenti riferimenti a Sterne; gli intenti spesso parodici («dénégation ludique», mescolanza «du satirique et du ludique»); infine «une pragmatique du badinage» che degrada un'idea di base, scientifica o politica, in divulgazione sensazionalista ed effimera.

La seconda parte, dedicata ai saggi di scrittori («La critique à l'essai»), è composta da un primo articolo di sébastien BAUDOIN (Chateaubriand essayiste dans l'“'Essai sur la littérature anglaise"), in cui l'A. rileva il carattere di mosaico letterario dell'opera, nata a ridosso della traduzione di Milton e costituita di osservazioni formulate in periodi diversi, nonché di pagine dei Mémoires, benché sostenuta da una solida «conscience structurante» che vede in primo piano la successione delle biografie dei «génies».

Dopo aver scelto, per tentare di definire Les Causeries di Sainte-Beuve, un approccio " externe», cioè formato dalle regole imposte al genere dallo stesso autore, Jean-Pierre BERTRAND (Peut-on définir la causerie? Le cas Sainte-Beuve) prende via via in esame le dichiarazioni programmatiche («Préface», annuncio di pubblicazione nel «Constitutionnel»), le testimonianze di persone e lettere e infine la ricezione critica, concludendo sul carattere di vero e proprio essai dell'opera di Sainte-Beuve.

7 Il rapporto fra essai e ironia è al centro della riflessione di michela LANDI su Baudelaire, La critique ou la vie. Baudelaire essayiste. Muovendosi fra i diversi scritti critici del poeta, con il supporto spesso della corrispondenza, l'A. mostra da un lato i meccanismi dell'ironia baudelairiana (l'uso della citazione e del corsivo, «l'annulation par ajout», l'«ironie syntagmatique»), dall'altro la forte polarizzazione fra il modello di Hugo e quello di Gautier, personificazione entrambi di una diversa «fonction» oggetto della mordente ironia di Baudelaire: «l'inspiration» e «le travail journalier».

8 Edyta COCIUBIN'SKA in L'essai sur le dandysme est-il un essai dandy? mostra come la chiave del successo del saggio di Barbey d'Aurevilly Du dandysme et de George Brummel stia precisamente nello stile seducente e appunto «dandy» dell'opera, trattato erudito che vira improvvisamente verso una imprevedibile e «insoutenable légèreté».

«Essais et fantaisie», terza parte, è inaugurata da Daniel SANGSUE con un saggio "trasversale" sull'influenza di Sterne via Hoffmann su alcune opere della letteratura francese del secondo Ottocento (Vie et opinions. L'essayisme sternien au XIX siècle). Da Vie et opinions de Tristram Shandy e Vie et opinions du Chat Murr Sangsue approda quindi a «Vie et opinions philosophiques d'un chat», racconto inserito nel Voyage aux Pyrénées di Taine, cui aggiunge, dello stesso autore, Vie et opinions de M. Frédéric-Thomas Graindorge. Nell'ultimo avatar sterniano, La Rôtisserie de la reine Pédauque di Anatole France, e nella sua continuazione Les Opinions de M. Jérôme Coignard la mediazione di Diderot si fa più evidente, senza sopraffare mai l'origine sterniana.

10 La relazione fra articolo giornalistico e saggio appare particolarmente interessante nel caso di Théophile Gautier, autore di circa 3000 articoli e di nessun «essai» definito come tale. Su questa base, Martine LAVAUD analizza in L'essai selon Théophile Gautier le pubblicazioni di Gautier in rivista, soprattutto i Grotesques («arbitraire révendiqué» e «goût pour le bizarre»), gli articoli nella "petite presse» (testi satirici e caricaturali) e nella «grande presse officielle» (particolarmente l'articolo su Gavarni del 1845).

11 L'essayisme nervalien di Henri SCEPI prende avvio dalla triplice accusa formulata da Nerval in Les Nuits d'octobre nei confronti del narratore: «Fantaisiste! réaliste!! essayiste!!!». Il 
realismo, invocato all'inizio del racconto, costituisce tuttavia per Scepi la zona specifica dell'«essayisme nervalien» frutto dell'«empirisme des rencontres et des contacts», ugualmente distante dall'«exactitude fantastique» e dall'«exactitude pédante». Procedendo per «diffraction», il racconto si alimenta di una discontinuità accidentale, nonostante la sua visibile coagulazione nella figura «logoscénique» dell'amico o nelle allusioni alla Divine Comédie, rivelando in realtà la materia autobiografica che lo compone: «l'écriture livre le sujet à ses lacunes, à ses angoisses et à ses fantasmes».

La quarta parte del volume, «Essais et savoirs: droit, histoire, philosophie» comprende tre contributi settoriali. L'essai dans la littérature juridique française $d u$ XIX ${ }^{e}$ siècle di Nader HAKIM riflette soprattutto sulle categorie utilizzate dai vari autori per classificare i saggi giuridici e sull'evoluzione diacronica del genere dalla seconda metà dell'Ottocento alla Belle Époque.

Rhétorique et histoire di Fabrice WILHELM propone alcune ipotesi sull'origine dei numerosi saggi, di argomento erudito o quotidiano, prodotti da Hippolyte Taine in applicazione della sua visione deterministica e del suo gusto polemico, ma anche in conseguenza dei numerosi studi filosofici e psicologici, nonché di quella che l'A. definisce la sua «imagination sympathique».

Alexandre DE VITRY propone infine una lettura in chiave politica dei saggi di Renan scritti dopo il 1870 (Renan, un éthos politique dans l'essai?). Da una posizione «de retrait permanent» e tuttavia sensibile alla collettività, l'éthos di Renan dopo il 1870 evolve secondo l'A. verso la concezione di una piccola comunità ideale, un gruppo di eletti che conferma la vocazione aristocratica di Renan, pur nell'interesse per il bene comune.

In «Formes de l'essai littéraire fin de siècle», quinta e ultima parte, si torna in ambito letterario con un contributo di Gaël PRIGENT, "Le Latin mystique", un essai littéraire? Trattato erudito, nutrito di numerosi riferimenti a diversi autori, il testo di Gourmont presenta tuttavia altri due elementi rilevanti: il commento personale delle parafrasi bibliche e la loro valutazione estetica. L'evidente attrazione per la lingua preziosa e rara dei mistici latini e il tentativo di tradurla attraverso una sorta di «transformation poétique» attenua, secondo l'A., la contrapposizione con Huysmans, che rimproverava alle traduzioni di Gourmont di essere al tempo stesso inesatte e inerti.

pierre V. ZIMA affronta in L'essayisme de Marcel Proust il delicato problema dello statuto della scrittura proustiana, definendo $L a$ Recherche «un roman essayiste». Più evidente nei saggi veri e propri, il carattere ambivalente della scrittura di Proust crea continuamente anche nel romanzo una tensione irriducibile fra l'associazione paradigmatica (segno dell'inconscio e del sogno) e la «structure essayiste» che cerca di ricostituire una fragile realtà evenemenziale. È ciò che Adorno, nei suoi «Petits commentaires de Proust», definisce con la nozione di constellation.

17 L'essai d'un portrait de l'intellect en tant que simulacre di cornelia KLETTKE chiude il volume con uno studio sull'Introduction à la méthode de Léonard de Vinci. Adottando la definizione di «essai conceptuel» proposta da Glaudes e Louette per i Cahiers, l'A. sottolinea il carattere esplorativo delle potenzialità umane che il saggio di Valéry presenta, costruito com'è sulla «verbalisation d'analogies à l'aide d'images» e dunque espressione di una logique imaginative, la cui continuità non è percepibile che da un intelletto come quello di Leonardo, dall'«homme tout intelligence». 